\title{
Adult size, longevity and fecundity in the seaweed fly, Coelopa frigida
}

\author{
R. K. Butlin* and T. H. Day
}

Department of Genetics, University of Nottingham, University Park, Nottingham, NG7 2RD.

An inversion polymorphism of chromosome $I$ in the seaweed fly, Coelopa frigida, is known to affect adult size. The relationship between adult size and components of fitness is, therefore, of particular interest. The longevity of males and females, female fecundity and the number of matings achieved by males have been measured using laboratory lines and have all been found to increase with size. The consequences for the maintenance of the inversion polymorphism are discussed.

\section{INTRODUCTION}

Large size is an advantage in a wide variety of ecological contexts. In particular, large females often have greater longevity and higher fecundity (see Hinton 1981, Vol I for examples among insect species), and large males usually have enhanced mating success (see Day et al., 1984). However, while there have been many studies of the relationship between size and fitness components, the heritability of size variation is known in only a few species (e.g., Drosophila, Robertson 1955) and so the evolutionary consequences of selection for large size cannot be assessed. Indeed, in many cases size is known to be strongly influenced by the environment, and so heritabilities may well be low.

In the seaweed fly Coelopa frigida, adult size is extremely variable, especially in males. Much of this variation is due to competition amongst larvae (Butlin and Day, 1984), but there is also an inherited component of variation, in males at least, associated with the $\alpha / \beta$ inversion polymorphism on chromosome I (Butlin et al., 1982). Under laboratory conditions large males have greater mating success than small males (Butlin et al., 1982). In more natural conditions, small males achieve more matings (Day et al., 1984), but in these experiments the effects of size and genotype effects were confounded. We report here observations on other components of fitness that may be

\footnotetext{
* Present address: School of Biological Sciences, University of East Anglia, Norwich, NR4 7TJ
}

influenced by adult size--namely longevity and fecundity.

\section{MATERIALS AND METHODS}

Newly eclosed flies were aspirated at random from four stock cultures: an inbred line, homokaryotypic for the $\alpha$ inversion sequence (designated $\alpha \alpha)$, one fixed for the $\beta$ sequence $(\beta \beta)$, the $F_{1}$ progeny of a cross between these lines $(\alpha \beta)$, and a relatively outbred $\beta \beta$ line ( $\beta \beta$ outbred). (Details of the derivation of these lines are given by Butlin 1983). Each female was given two new males in a $7 \times 2 \mathrm{~cm}$ vial of fresh culture medium every day until it died. The eggs in each egg batch laid were counted, and retained for $48 \mathrm{hrs}$ to check for hatching. To measure male fecundity, individual males were each given two virgin females in a vial of fresh medium every day until the male died. The two females were retained together in their vial for three days, and the appearance of larvae in the vial was then taken to indicate that the male had mated with at least one of the females. All flies were kept in constant darkness at $27^{\circ} \mathrm{C}$. The sizes of flies were estimated from their wing lengths and are expressed in graticule divisions where 1 division $=1.45 \mathrm{~mm}$ (Butlin et al., 1982).

\section{RESULTS}

In both males and females, the flies from the four lines had broadly overlapping size distributions 
but different mean sizes. It is therefore impossible to separate completely line effects from size effects. We have taken the conservative approach of testing for line differences after fitting regressions on size and for a regression on size, after fitting line differences for each character.

Consider longevity first. For females (fig. la) there was a significant difference between lines $\left(F_{3,73}=5.26, \quad P<0.01\right)$, but no significant regression on size after allowing for line differences $\left(F_{1,73}=3 \cdot 39,0 \cdot 10>P>0 \cdot 05\right)$. For males (fig. $\left.1 \mathrm{~b}\right)$, both effects were highly significant (for line differences $F_{3,75}=6.97, P<0.001$; for regression on size $\left.F_{1,75}=11 \cdot 22, P<0 \cdot 01\right)$. In both sexes, $\alpha \beta$ flies were the largest and the longest lived, but there was less variation in size in the females. It seems that, in general, large flies live longer but that there are also line-specific effects which are independent of size.

The two measures of female fecundity both showed a very strong influence of size (figs $2 a$ and $2 b)$ : for the number of egg batches $F_{1,70}=31 \cdot 49$, $P<0.001$; for the mean number of eggs per batch $F_{1,59}=65 \cdot 68, P \ll 0 \cdot 001$. The increase in number

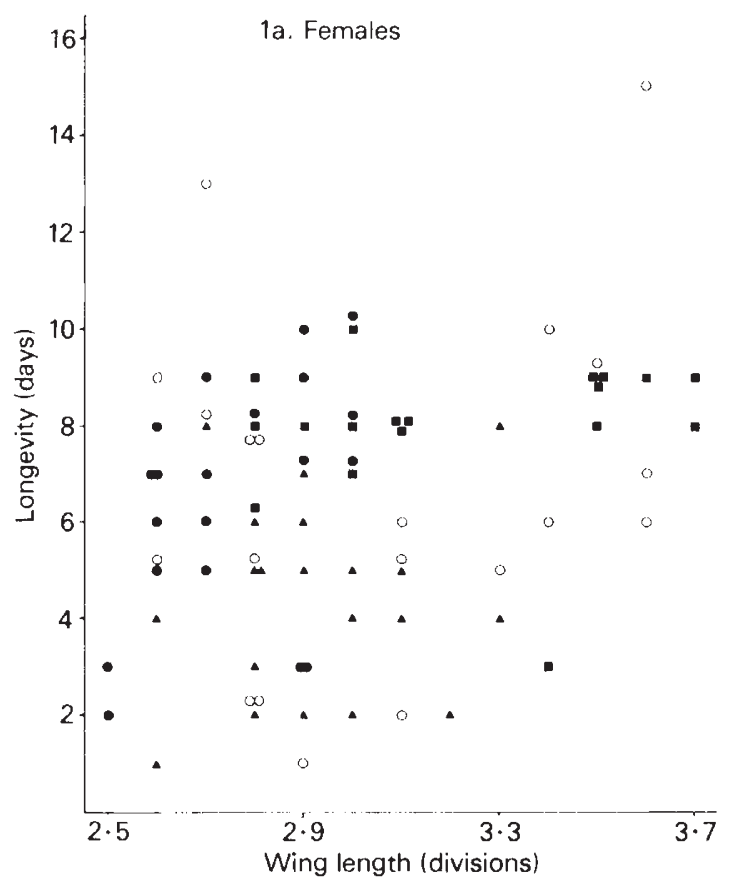

Figure 1 The relationship between wing length and longevity for a) females $(b=1.95 \pm 1.06)$ and b) males $(b=2 \cdot 19 \pm$ $0.65)$.

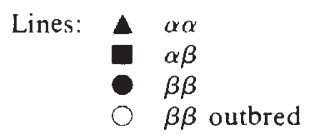

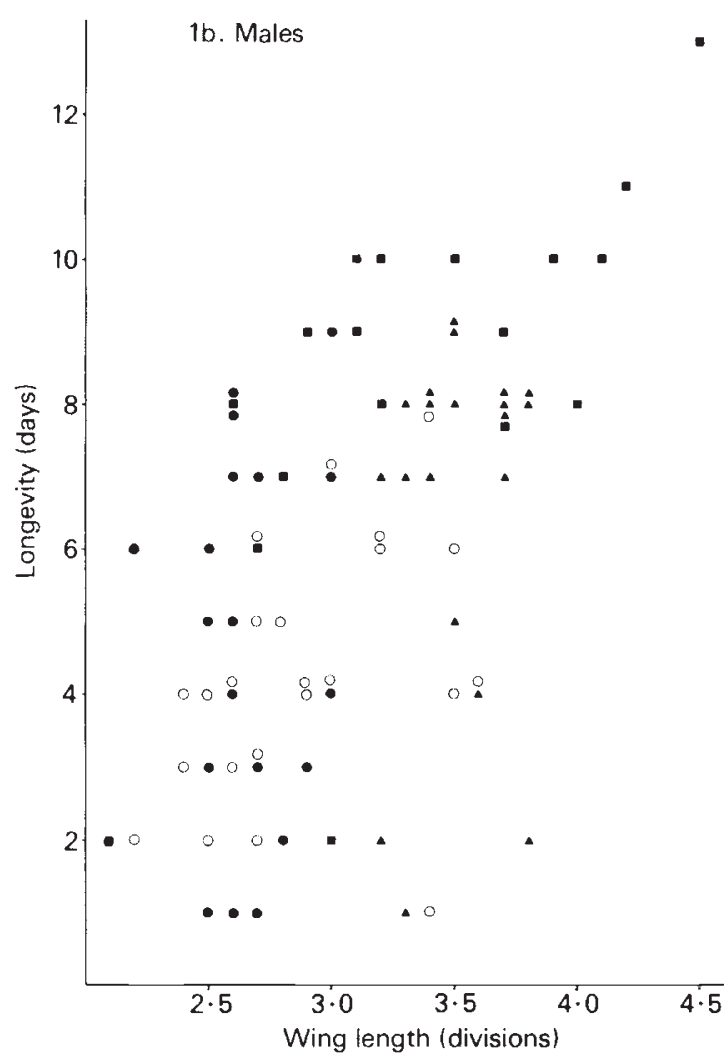

of egg batches clearly results from the effect of size on longevity - most females laid an egg batch every two or three days. Only when the number of eggs per batch is considered, was there a line difference $\left(F_{3,59}=3 \cdot 80, P<0 \cdot 05\right)$-mainly due to the greater number of batches laid by the $\beta \beta$ outbred line.

Male fecundity was measured as the number of days on which a male successfully fertilised at least one female (fig. 3 ). This is a rough minimum estimate, but nevertheless it showed a marked increase with size $\left(F_{1.75}=11 \cdot 72, P<0.01\right)$ as well as a difference between lines $\left(F_{3,75}=9 \cdot 80, P<\right.$ $0.001)$. The size effect can be explained simply as a result of increased longevity but the line differences cannot. Analysing the proportion of days (transformed to angles) on which a female was fertilised, the regression on size was not significant $\left(F_{1,75}=1 \cdot 16, P>0 \cdot 20\right)$, but the line differences were $\left(F_{3,75}=6 \cdot 11, P<0 \cdot 001\right)$. It is, perhaps, surprising that there were any days on which males failed to inseminate females. It is possible that female preferences as well as male fecundity may be involved. If females consistently reject some males even in the absence of alternative mates, this would imply a female preference for large absolute male size, or large size relative to their own size. 
However Butlin et al., (1982) only detected an increase in mating success for the larger of two males. Male seaweed flies can fertilise at least 10 females in one day (Day and Barrand, unpublished results) so obviously a much larger experiment is needed to obtain a more reliable measure of male fecundity.

\section{DISCUSSION}

In common with many other insects, Coelopa frigida shows an increase in longevity and fecundity with adult size. In females the overall increase in egg output is very marked indeed, whereas in males the influence on reproductive success is more difficult to assess. The longer adult life of larger males, and possibly their greater mating frequency,

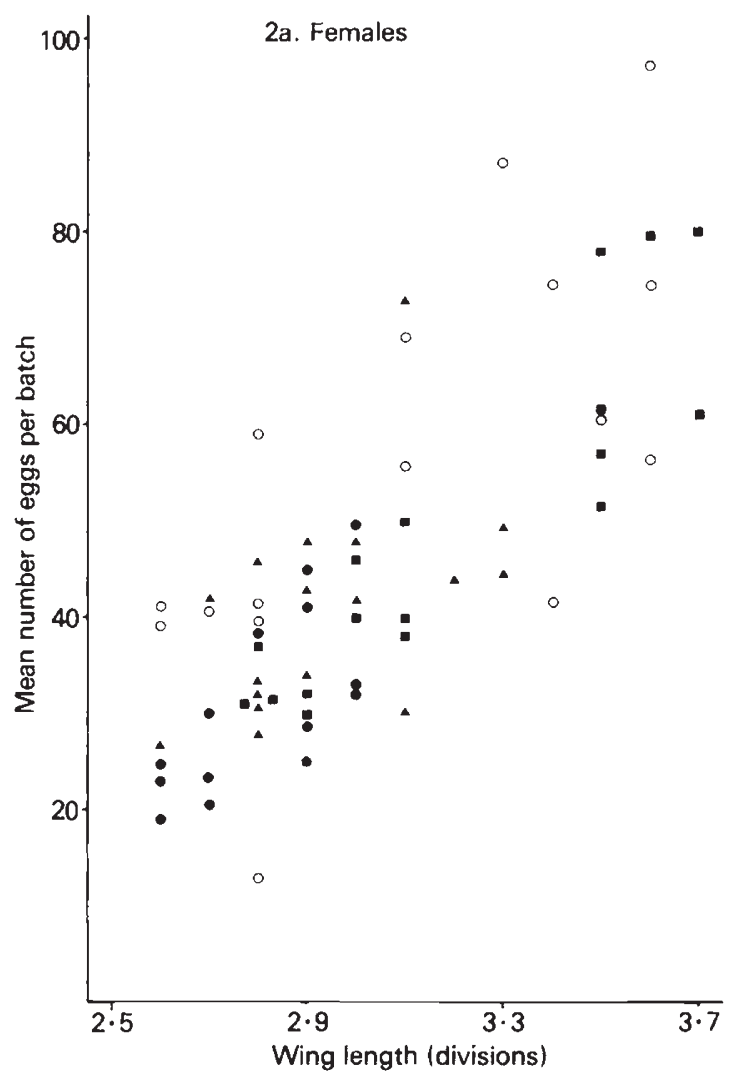

Figure 2 The relationship between wing length and fecundity of females: a) mean number of eggs per batch $(b=$ $39.71 \pm 4.90)$ and b) number of egg batches $(b=2.67 \pm$ $0 \cdot 48)$.

Lines:

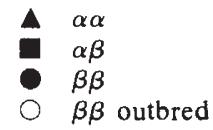

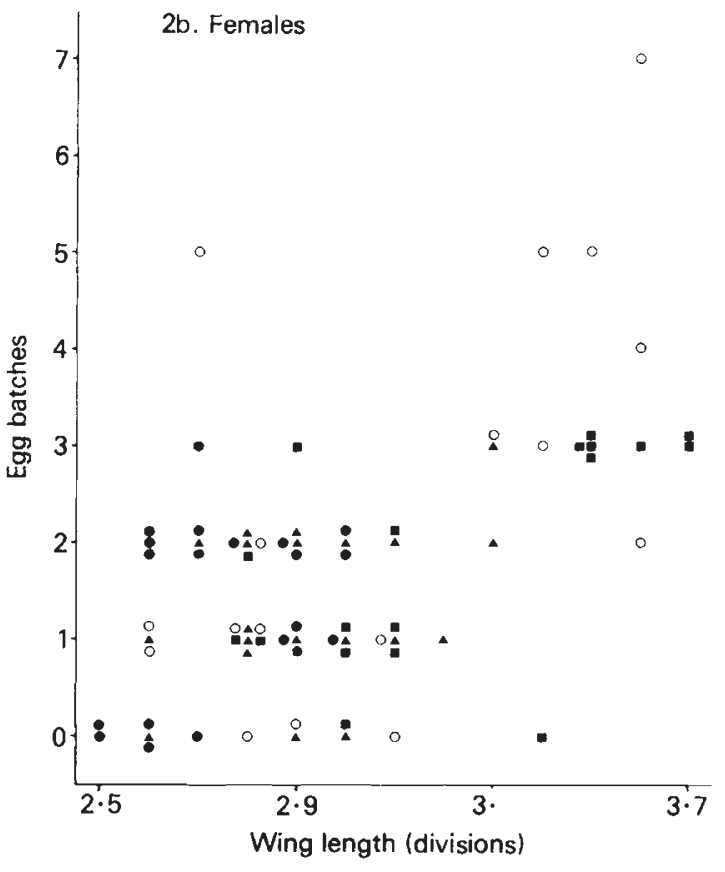

give them the potential to fertilise more females, but whether or not this will be realised depends on male-male interactions as well as those between males and females.

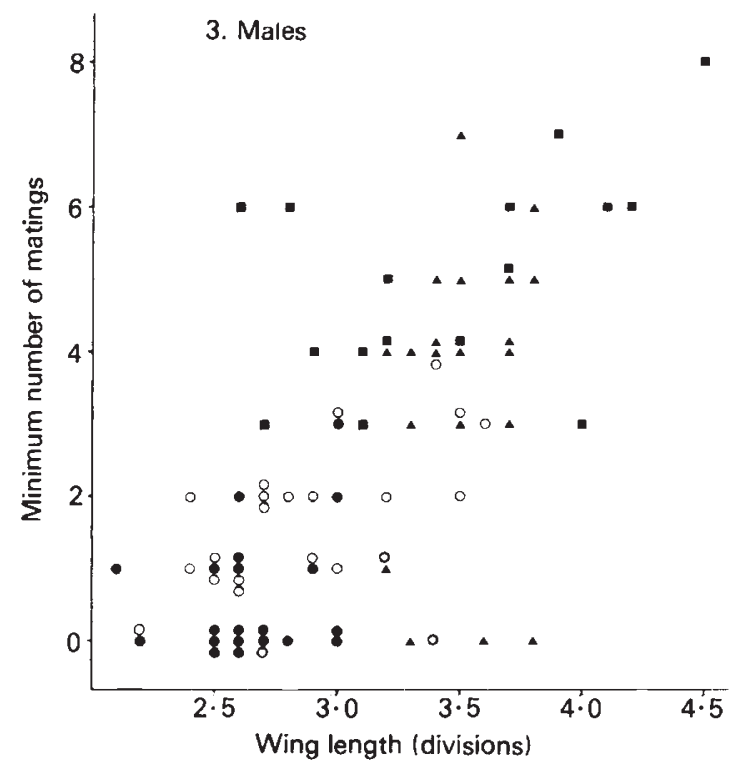

Figure 3 The relationship between wing length and minimum number of matings achieved by males $(b=1.46 \pm 0.43)$.

Lines:

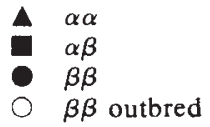


Two features of seaweed flies make these observations of particular interest. First, there is a heritable component to size associated with the $\alpha / \beta$ inversion system. In a typical natural population $\alpha \alpha$ females have wing lengths about $0.3 \mathrm{~mm}$ (7 per cent) longer than $\beta \beta$ females (Butlin et al., 1982). The present results suggest that this is associated with an increase in longevity of about 0.5 days, and a rise in total egg production of about 40 eggs (about 25 per cent). In males the typical size difference is much greater $-\alpha \alpha$ males are about $1.5 \mathrm{~mm}$ ( 25 per cent) longer than $\beta \beta$ malesand in these experiments this size difference corresponds to a doubling of both longevity and number of matings. The large size of $\alpha \alpha$ and $\alpha \beta$ flies clearly represents a considerable selective advantage over $\beta \beta$ flies.

The line differences observed in these experiments cannot be taken as evidence for size independent karyotypic effects. They may be due to other genetic differences between the inbred lines or to differences in rearing conditions. However, the fact that the $\alpha \beta$ and $\beta \beta$ outbred lines tended to do best may be because they suffered less from inbreeding.

The second feature of interest in the Coelopa system is that a selective force acting in opposition to the advantage of large size is known. At any larval density large flies take longer to develop than small flies (Butlin and Day 1984). Rapid development is essential for Coelopa because it lives in piles of rotting seaweed that are periodically swept out to sea by high tides and storms. The advantage of large size must, therefore, be balanced against the increased risk associated with long development time.

\section{REFERENCES}

BUTLIN, R. K. 1983. The maintenance of an inversion polymorphism in Coelopa frigida. PhD Thesis, University of Nottingham.

BUTL.IN, R. K. AND DAY, T. H. 1984. The effect of larval competition on development time and adult size in the seaweed fly, Coelopa frigida. Oecologia, 63, 122-127.

BUTLIN, R. K., READ, I. L. AND DAY, T. H. 1982. The effects of a chromosomal inversion on adult size and male mating success in the seaweed fly, Coelopa frigida. Heredity, 49, $51-62$.

DAY, T. H., MILES, S., PILKINGTON, M. AND BUTLIN, R. K. 1984. Sexual selection in seaweed flies. Submitted to Heredity.

HINTON, H. E. 1981. The Biology of Insect Eggs. Pergamon Press, Oxford.

ROBERTSON, F. W. 1955. Selection response and the properties of genetic variation. Cold Spring Harb. Symp. Quant. Biol., 20, 166-177. 